\title{
Profesionalismo y el Cambio de Paradigma en los Métodos de Evaluación de Educación Médica.
}

\section{Professionalism and the paradigm shift in assessment methods in medical education.}

\author{
Kenneth R. Fernández Taylor
}

Estudiante de $5^{\circ}$ año de Medicina, Facultad de Ciencias de la Salud Dr. Luis Edmundo Vásquez, Universidad Dr. José Matías Delgado, La Libertad, El Salvador.

Palabras clave: Educación médica, profesionalismo, médicos en formación. Key words: Medical education, professionalism, physicians in training.

"La forma de la evaluación que se haga determina no solo la forma en que se estudia y se adquieren diferentes destrezas, sino que determina incluso la calidad de la vida académica del docente y del estudiante por igual! La evaluación no debe frustrar ni destruir a quien la reciba".

Jose Venturelli, Universidad de McMaster, Canada ${ }^{1}$.

El profesionalismo incluye no solo conocimientos y habilidades médicas sino que también la ética, el desarrollo de habilidades de comunicación y relaciones interpersonales, compasión, pensamiento crítico, base científica, etc ${ }^{2}$; por lo tanto, siendo la medicina una disciplina multidimensional, el proceso de aprendizaje en medicina requiere más que el desarrollo de la capacidad de memorización. Algunos se preguntarán que tienen que ver los métodos de evaluación con el profesionalismo; la respuesta es: mucho, ya que lo que se evalúa y el método que se utiliza para hacerlo determina las habilidades y el conocimiento que el estudiante adquiere.

En la última década hemos observado la rápida evolución de las metodologías de evaluación en educación médica desde los métodos tradicionales hasta las estrategias de evaluación más sofisticadas. Además, a nivel mundial se realizan esfuerzos para establecer estándares internacionales de calidad sobre el área en cuestión ${ }^{3}$ (ver artículo pág. 14 de está edición). El uso de métodos de evaluación como el Objective Structured Clinical Evaluation (OSCE), exámenes escritos estandarizados, pacientes simulados, entre otros, han pro-

\footnotetext{
Correspondencia:
}

Kenneth Fernández

Facultad de Ciencias de la Salud, Dr. Luis Edmundo Vásquez Universidad Dr. José Matías Delgado. La Libertad, El Salvador e-mail: kf2013@hotmail.com vocado un gran efecto en el amplio sistema de educación médica y en la profesión médica en general ${ }^{4}$. El slogan comúnmente utilizado "la evaluación le da dirección al aprendizaje", aunque cierto, representa un concepto cerrado, por lo que se decidió cambiarlo por el de "la evaluación expande los horizontes profesionales" (M. Friedman, 2000). Esto enfatiza el rol de la evaluación en el desarrollo de las múltiples dimensiones que abarca la profesión médica.

\section{EL CAMBIO DE PARADIGMA}

El cambio de paradigma se refiere precisamente al progresivo desplazamiento de los tradicionales métodos cuantitativos de evaluación por métodos formadores como el OSCE que premian otras habilidades en los estudiantes y desacreditan la puramente memorística que en los programas tradicionales son del $30 \%$ y el $70 \%$ respectivamente, en el mejor de los casos, evidenciándose excesivo énfasis en lo que los expertos han llamado "rote learning" 5 . El cambio de paradigma ha sido de evaluar conocimiento teórico a evaluar competencia en el ambiente de trabajo.

Lo importante es el método, no la regurgitación de cantidades masivas de materia fáctica. Lo que es memorizado rápidamente se olvida, se desactualiza, mientras que la retroalimentación de un maestro en el 
escenario clínico es algo que pudiera no olvidarse para toda la vida. Sir George Alleyne, dijo hace algunos años: "los aspectos de la evaluación, que determinan la forma de estudiar han sido simplemente ignorados y se han mantenido sistemas que hacen del estudiante un pasador de exámenes y no una persona que va a responder a las necesidades de un país en un área determinada". Los expertos concuerdan en que "para saber si un estudiante es competente necesitamos observar al estudiante trabajando in vivo no en aislamiento in vitro bajo condiciones de examen" ${ }^{6}$. Esto en nuestro medio se vuelve aún más importante, ya que en diversas circunstancias, por falta de médicos en el hospital, los estudiantes deben trabajar sin ninguna supervisión.

\section{DISOCIACIÓN ENTRE TEORÍA Y PRÁCTICA}

Es importante hacer notar que los exámenes escritos y pruebas de elección múltiple, que ejercitan únicamente el lado izquierdo del cerebro (y que por supuesto no pueden ser eliminados del todo), no capacitan al estudiante para situaciones con pacientes reales; no le capacitan sobre como es que debe interactuar con los distintos elementos del equipo de personal hospitalario, ni mucho menos sobre como debe él entablar una buena comunicación con sus pacientes. Es cierto, se envía a los estudiantes a "turnos" a los hospitales, donde si tienen suerte se encontrarán algún Residente altruista quien logre hacer el tiempo (que en los hospitales públicos es muy escaso) para darle dirección a los médicos en formación. El problema aquí es que en muchos casos los estudiantes no reciben dirección de nadie, ya que durante la noche o fines de semana, que es cuando se hacen los turnos, no hay ningún elemento del staff de los hospitales, es decir, ningún médico ya formado. La realidad por sí sola no es didáctica ${ }^{7}$.

\section{¿QUIÉN Y CUÁNDO EVALÚA LA ADQUISICIÓN DE DESTREZAS CLÍNICAS?}

No es que los estudiantes no tengan nada en que ocuparse; de quererlo siempre encuentran algo que hacer; pero ¿estarán haciendo bien lo que hacen? ¿Serán ellos capaces de identificar todas las destrezas que deberían aprender? ¿Aparte de aprender la técnica para poner una sonda vesical, aprenderá el estudiante lo mínimo durante la rotación de Medicina Interna sobre las cualidades de profesionalismo y comunicación que requerirá para el ejercicio de su profesión? ¿Estarán los pacientes a salvo a las 3:30 a.m. en manos de un "Externo" inexperto y agotado? Nadie lo sabe, pero la lógica apunta que sin una buena guía todo barco se va a la deriva. Más aún, en un ambiente como el de nuestros hospitales. Al séptimo año, el estudiante llegará al Internado y allí, este preparado o no, deberá asumir de una forma u otra todas las responsabilidades que el cargo confiere que con frecuencia son de vida o muerte. Es por esto que es importante cultivar el profesionalismo a través del cambio de paradigma en los métodos de evaluación en educación médica.

Una revisión del Centro para el Desarrollo del Staff en Oxford identificó cinco aspectos de los programas educacionales en el currículum de medicina de pregrado que se asocian con que los estudiantes adopten técnicas de aprendizaje indeseables, superficiales, que les imposibilitan la obtención de los principios fundamentales de un tema específico. Los cinco aspectos son: Sobrecarga laboral, sobrecarga académica, poca oportunidad de estudiar las asignaturas a profundidad, poca opción de escoger los tópicos y métodos de estudio, y sistemas de evaluación provocadores de ansiedad que premian o toleran la regurgitación de información fáctica.

Kevelighan, Duffy, Walker. Innovations in teaching obstetrics and gynaecology - the Theme Afternoon. Medical Education 1998 32:5 p. 517

Algo muy similar fue detectado el año pasado y presentado a las autoridades académicas por los Estudiantes de Medicina de la ASESMED de la Facultad de Ciencias de la Salud Dr. Luis Edmundo Vásquez tras la elaboración del documento "Propuestas para el mejoramiento de la educación médica en la FACSALEV".

\section{BIBLIOGRAFÍA}

1. Venturelli J. Educación Médica: Nuevos Enfoques, Metas y Métodos. OPS/OMS. Washington D.C. 1997.

2. Wojtczak A. Evaluation of Learning Outcomes: Assessment Methods and Measurement. Working Review, August 2000. http://www.iime.org

3. World Federation for Medical Education. Basic Medical Education. WFME Global Standards for Quality Improvement. Copenhagen: WFME 2003. http://www.wfme.org

4. Ogburn, Tony MD. Espey, Eve MD. The R-I-M-E method for evaluation of medical students on an obstetrics and gynecology clerkship. American Journal of Obstetrics \& Gynecology. 189(3):666-669, September 2003.

5. Regan-Smith, Martha G. MD, EdD. Et al. Rote Learning in Medical School. JAMA, Volume 272 (17), 1994. pp 1380-1381.

6. Wojtczak A. Op cit.

7. Nolla Domnejó, María. Aprendizaje y Contextos Reales. Educación Médica 2002; 5(4):161-2. 